(12) and the boundary conditions established above, $u+v$ has the constant value -1 along $A^{\prime} C$, and $u-v$ has the constant value 1 along $B^{\prime} C$. At $C$, we have therefore

$$
u=0, \quad v=-1 \text {. }
$$

Equations (21) show that the entire region $D O E$ moves as a rigid body with unit speed in the negative $y$ direction. Similarly, $D^{\prime} O E^{\prime}$ moves as a rigid body with unit speed in the positive $y$ direction, and the instantaneous plastic flow is restricted to the lines of discontinuity $D D^{\prime}$ and $E E^{\prime}$.

On account of the great simplicity of this velocity field, finite plastic deformation is readily discussed. Figure 2 shows the specimen in a later stage. Plastic deformation has occurred only in the shaded portions; the unshaded portions have moved as rigid bodies. At the considered instant, all points to the right of $D D^{*} O^{*} E^{* \prime} E$ move with unit speed in the position $x$ direction, all points above $E^{*} O^{*} D^{*}$ move with unit speed in the negative $y$ direction, etc., the instantaneous plastic flow being again restricted to the lines of discontinuity $D^{*} D^{* \prime}$ and $E^{*} E^{* \prime}$. Since the arc $D^{*} E^{*}$ in Fig. 2 is congruent with the central part of the arc $D E$ in Fig. 1, the stresses in the region $D^{*} O^{*} E^{*}$ are given by the equations obtained from (19) by substitut-

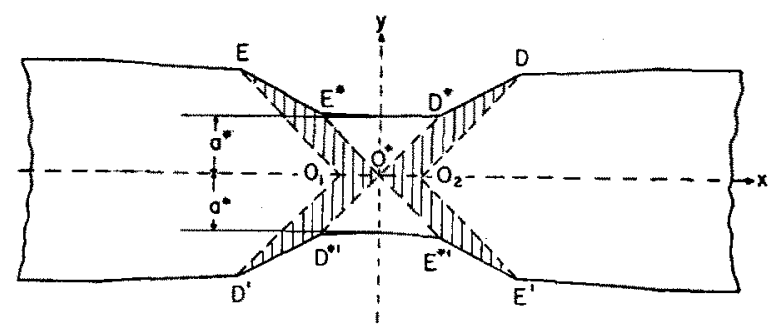

FIG. 2.

ing $a^{*}$ for $a$. If the considered necking process continues without rupture, the central portion of the specimen will eventually consist of two wedges with common apex. If the initial grooves were rather shallow, these wedges will have practically rectilinear sides that have the slope $1: 2$ with respect to the $x$ axis.

It is well known (see, for instance, reference 6, p. 251) that the plastic stress and velocity fields considered here are possible only if the initial grooves are "sufficiently deep." The precise criterion for "sufficient depth" is not known. For the parabolic groove specified by (13), the usual estimate of the necessary depth leads to the statement that the length of the groove should be at least $4 a$. This condition is fulfilled in Fig. 1.

\title{
The Growth of Vapor Bubbles in Superheated Liquids*
}

\author{
M. S. Plesset and S. A. Zwick \\ California Institute of Technology, Pasadena, California
}

(Received September 26, 1953)

\begin{abstract}
The growth of a vapor bubble in a superbeated liquid is controlled by three factors: the inertia of the liquid, the surface tension, and the vapor pressure. As the bubble grows, evaporation takes place at the bubble boundary, and the temperature and vapor pressure in the bubble are thereby decreased. The heat inflow requirement of evaporation, however, depends on the rate of bubble growth, so that the dynamic problem is linked with a heat diffusion problem. Since the heat diffusion problem has been solved, a quantitative formulation of the dynamic problem can be given. A solution for the radius of the vapor bubble as a function of time is obtained which is valid for sufficiently large radius. This asymptotic solution covers the range of physical interest since the radius at which it becomes valid is near the lower limit of experimental observation. It shows the strong effect of heat diffusion on the rate of bubble growth. Comparison of the predicted radius-time behavior is made with experimental observations in superheated water, and very good agreement is found.
\end{abstract}

\section{INTRODUCTION}

W HEN the vapor pressure in a liquid exceeds the ambient pressure, it becomes possible for a vapor bubble to grow from a small "nucleus" in the liquid. This nucleus is a region of nonliquid phase and presumably consists of a gas or vapor phase stabilized on a solid particle. The rate of growth of a vapor bubble, once formed, is determined by the surface tension, the

\footnotetext{
* This study was supported by the U. S. Office of Naval Re-
}

liquid inertia, and the difference between the pressure within the bubble and the ambient, or external, pressure. In the initial stages of the bubble expansion, when the forces are nearly in equilibrium, the growth is slow but it is accelerated with increase in bubble size because of the reduction in surface tension. When the rate of bubble growth becomes appreciable, however, the temperature and, hence, the pressure within the bubble drop and the rate of growth is decreased. One might, therefore, expect a maximum in the velocity of the bubble wall. The reduction of the temperature 
within the bubble is a consequence of the latent heat requirement of the evaporation which takes place at the vapor-liquid interface as the bubble grows.

For the quantitative solution of the problem, some simplifying physical assumptions may be made. The example considered in detail here is that of a vapor bubble growing in moderately superheated water, and the arguments for the validity of the general assumptions are justified for this case.

It will be assumed that the bubble is spherical throughout its growth. This assumption is reasonable provided the radial acceleration and velocity are small, for with a spherically symmetric external pressure field the spherical shape will then be stable under the action of surface tension. ${ }^{1}$ Excluded from consideration is the asymmetric, buoyant force of gravity which becomes important if the bubble growth is followed for so long a time that a significant translational velocity is acquired. A translational velocity of the bubble as a whole not only causes a deformation in shape but also increases the rate of heat inflow to the bubble over that used in the analysis presented below. In water superheated by about $10^{\circ} \mathrm{C}$, no great error is introduced by the buoyant force provided the bubble growth is not followed beyond a radius of approximately $1 \mathrm{~mm}$.

For a superheat of $10^{\circ} \mathrm{C}$ in water, a vapor bubble grows from its initial microscopic size to a radius of 1 $\mathrm{mm}$ in a time of the order of $10 \mathrm{msec}$. The corresponding average radial velocity of $10 \mathrm{~cm} / \mathrm{sec}$ is very small compared with the velocity of sound in the liquid, so that compressibility effects may be entirely neglected in the motion of the liquid produced by the bubble growth. It may also be shown that viscous effects are unimportant. ${ }^{2}$ The hydrodynamic equation of motion, which contains the effect of the liquid inertia, is thus greatly simplified. The bubble wall velocity is also very small compared with the velocity of sound in the vapor so that compressibility effects are unimportant here as well. One has the further simplification in the vapor region that the vapor density is so small that its inertia effects may be neglected. It then follows from Bernoulli's equation applied to a fluid of negligible density that the pressure within the vapor region may be taken as uniform. In addition, since the acoustic velocity in the vapor is so large, the pressure within the vapor follows practically instantaneously its value at the bubble wall. When the velocity of the bubble wall is sufficiently slow, the pressure of the vapor is given by the equilibrium vapor pressure of the liquid. That this is the case for the present problem may be seen as follows. The mean velocity appropriate for the rate of evaporation from a liquid surface is $(B T / 2 \pi M)^{\frac{1}{2}}$, where $B$ is the gas constant, $T$ the absolute temperature, and $M$ is the molecular weight. This velocity must be reduced by the coefficient for

\footnotetext{
1 M. S. Plesset, J. Appl. Phys. 25, 96 (1954).

2 F. R. Gilmore, Report 26-4, Hydrodynamics Laboratory, California Institute of Technology, April 1952.
}

evaporation which has the value 0.04 for water. ${ }^{3}$ The critical velocity for a water surface at approximately $100^{\circ} \mathrm{C}$ is therefore about $8 \mathrm{~m} / \mathrm{sec}$, which is appreciably greater than the radial velocities encountered here, so that the pressure deficiency from the equilibrium vapor pressure may be neglected.

The temperature of the vapor would, in general, vary with position in the bubble as well as with time. The approximation will be made, however, that the thermal diffusivity $D^{\prime}$ of the vapor is so large that temperature gradients are negligible within the bubble. In water vapor, the characteristic diffusion length $\left(2 D^{\prime} t\right)^{\frac{1}{3}}$ is $0.24 \mathrm{~cm}$ for $t=10^{-2} \mathrm{sec}$. For the greatest superheat considered here $\left(\approx 10^{\circ} \mathrm{C}\right)$ the bubble radius at this time is approximately $0.1 \mathrm{~cm}$, which is significantly smaller than the characteristic diffusion length. The approximation of uniform temperature within the vapor improves with decrease in superheat.

In summary, the physical model upon which the calculations are based consists of a spherical vapor bubble which has uniform temperature and pressure; the temperature of the vapor is that of the liquid at the bubble wall, and the pressure is the equilibrium vapor pressure for that temperature. In addition, the effects of viscosity and compressibility are neglected both in the vapor and in the liquid.

The equation of motion for the radius $R$ of the bubble in a nonviscous, incompressible liquid as a function of time is ${ }^{4}$

$$
R\left(d^{2} R / d t^{2}\right)+\frac{3}{2} \dot{R}^{2}=\left[p(R)-P_{0}\right] / \rho,
$$

where $\rho$ is the liquid density, $P_{0}$ is the external pressure in the liquid or the pressure at infinity, and $p(R)$ is the pressure in the liquid at the bubble boundary. $A$ dot denotes differentiation with respect to time. The pressure $p(R)$ is given in terms of the vapor pressure $p_{v}$ within the bubble by

$$
p(R)=p_{v}(T)-2 \sigma / R,
$$

where $\sigma$ is the surface tension constant and $p_{v}(T)$ is the equilibrium vapor pressure for the temperature $T$ at the bubble boundary. In the following, the small variations of $\sigma$ and $\rho$ with temperature are neglected. It is convenient to introduce a radius $R_{0}$ defined by the relation

$$
2 \sigma / R_{0}=p_{v}\left(T_{0}\right)-P_{0}
$$

in which $T_{0}$ is the temperature of superheat of the liquid at a distance from the bubble. Physically, $R_{0}$ is the effective initial radius of the bubble; it represents an extrapolation of the free spherical bubble down to the equilibrium radius for the given initial conditions. It should be noted that a bubble at rest with radius $R_{0}$ is in unstable equilibrium. The actual nucleus from which the bubble grows is not necessarily spherical and its surface energy may be appreciably less than $4 \pi \sigma R_{0}{ }^{2}$;

\footnotetext{
${ }^{2}$ G. Wyllie, Proc. Roy. Soc. (London) A197, 383 (1949).

G. Lamb, Bydrodynamics (Dover Publications, New York, 1945); M. S. Plesset, J. Appl. Mechanics 16, 277 (1949).
} 
however, the nucleus from which the bubble grows and the free spherical bubble of radius $R_{0}$ are both in unstable equilibrium at the temperature $T_{0}$ and external pressure $P_{0}$. Table I gives a set of values of $R_{0}$ at various superheat temperatures in water for a pressure $P_{0}$ of one atmos.

In terms of the parameter $R_{0}$, Eq. (1) may be written

$$
\begin{aligned}
R\left(d^{2} R / d t^{2}\right)+ & \frac{3}{2} \dot{R}^{2} \\
& =\frac{p_{v}(T)-p_{v}\left(T_{0}\right)+\left(2 \sigma / R_{0}\right)\left(1-R_{0} / R\right)}{\rho},
\end{aligned}
$$

or

$$
\begin{aligned}
\frac{1}{2 R^{2} \dot{R}} \frac{d}{d t}\left(R^{3} \dot{R}^{2}\right) & \\
= & \frac{p_{v}(T)-p_{v}\left(T_{0}\right)+\left(2 \sigma / R_{0}\right)\left(1-R_{0} / R\right)}{\rho} .
\end{aligned}
$$

If the cooling effect of evaporation is disregarded, so that $p_{v}(T)=p_{v}\left(T_{0}\right)$, Eq. (5) may be integrated to give

$$
\begin{aligned}
\dot{R}^{2}=\left(R_{0} / R\right) \dot{R}_{0}{ }^{2}+\left(4 \sigma / 3 \rho R_{0}\right) & {\left[1-R_{0}{ }^{3} / R^{3}\right] } \\
& -(2 \sigma / \rho R)\left[1-R_{0}{ }^{2} / R^{2}\right] .
\end{aligned}
$$

This solution will be referred to as the Rayleigh solution. ${ }^{6}$ For $R \gg R_{0}$, Eq. (6) becomes

$$
\dot{R}^{2} \sim \frac{4 \sigma}{3 \rho R_{0}}=\frac{2}{3 \rho}\left[p_{v}\left(T_{0}\right)-P_{0}\right]
$$

which is a constant.

The actual motion deviates markedly from that predicted by the Rayleigh solution because of the cooling effect. The heat $Q$ which must be supplied to the bubble per unit time is

$$
\dot{Q}=(4 \pi / 3) L(d / d t)\left(R^{3} \rho^{\prime}\right),
$$

where $L$ is the latent heat of evaporation per unit mass and $\rho^{\prime}$ is the vapor density. This heat is supplied by conduction from the liquid into the bubble so that

$$
\dot{Q}=4 \pi R^{2} k(\partial T / \partial r)_{R},
$$

where $k$ is the thermal conductivity of the liquid and $(\partial T / \partial r)_{R}$ is the temperature gradient in the liquid at the bubble boundary. Hence,

$$
(\partial T / \partial r)_{R}=(L / 3 k)\left(1 / R^{2}\right)(d / d t)\left(R^{3} \rho^{\prime}\right) .
$$

The small variations of $L$ and $k$ with temperature are neglected. It may be shown that the contribution of the term proportional to $d \rho^{\prime} / d t$ is much less than that from the term proportional to $d R^{s} / d t$. Equation (10) may be approximated, therefore, by

$$
(\partial T / \partial r)_{R} \approx\left(L \rho^{\prime} / k\right) \dot{R} .
$$

- The solution for the motion of a bubble under such constant pressure conditions was given by Rayleigh, Phil. Mag. 34, 94 (1917) and applied by him to the case of a collapsing bubble.
TABLE I. Effective nucleus size $R_{0}$ in water at one atmosphere for the values of the temperature $T_{0}$.

\begin{tabular}{lrrrrr}
\hline \hline$R_{0}\left(\mathrm{~cm} \times 10^{3}\right)$ & 1.56 & 1.02 & 0.751 & $\begin{array}{c}0.590 \\
105\end{array}$ & $\begin{array}{c}0.483 \\
106\end{array}$ \\
\hline \hline
\end{tabular}

This approximation is plausible physically since the volume increases by orders of magnitude, while the vapor density changes very little because of the small temperature decrease during the bubble growth.

Equation (11) together with the specification of the temperature at infinity $T_{0}$ determines the temperature field in the liquid. The solution for this temperature problem with the moving boundary $R(t)$ has been found ${ }^{6}$ under the assumption that the drop in temperature from $T_{0}$ to the value $T$ at the bubble boundary takes place in a layer of liquid adjacent to the bubble which has small thickness compared with $R(t)$. This approximation of the "thin thermal boundary layer" is justified physically because the thermal diffusivity of the liquid is small. The approximate expression for the temperature at the bubble wall is ${ }^{7}$

$$
T=T_{0}-\left(\frac{D}{\pi}\right)^{t} \int_{0}^{t} \frac{R^{2}(x)(\partial T / \partial r)_{r}=R(x)}{\left\{\int_{x}^{t} R^{4}(y) d y\right\}^{\frac{1}{2}}} d x .
$$

The temperature $T$ is thus given in terms of $R$ and $\dot{R}$ by Eq. (12) so that $p_{v}(T)$ is also specified in terms of these variables. Equations (5) and (12) therefore determine the dynamic problem of the bubble growth.

\section{SOLUTION OF THE EQUATION OF MOTION}

Equations (5) and (12) are connected when the equilibrium vapor pressure is specified as a function of temperature. For superheats not too far above the boiling temperature $T_{b}$ of the liquid at the external pressure $P_{0}$, the vapor pressure may be approximated by a linear function of the temperature,

$$
\frac{p_{v}(T)-P_{0}}{\rho}=A\left(T-T_{b}\right)
$$

For $T=T_{0}$, Eq. (13) gives

$$
2 \sigma / \rho R_{0}=A\left(T_{0}-T_{b}\right)
$$

because of Eq. (3). Equation (5) may now be written as

$$
\frac{1}{2 R^{2} \dot{R}} \frac{d}{d t}\left(R^{3} \dot{R}^{2}\right)=A\left(T-T_{0}\right)+\frac{2 \sigma}{\rho R_{0}}\left(1-\frac{R}{R_{0}}\right) .
$$

- M. S. Plesset and S. A. Zwick, J. Appl. Phys. 23, 95 (1952).

7 Reference 6, Eq. (20). The error in Eq. (20) may be estimated from $\mathrm{Eq}$. (31) of this reference. For the problem discussed here it was found to be less than 10 percent of the difference $T-T_{0}$ at any time. 
It is convenient to use in place of $R$ the dimensionless volume ratio

$$
p=R^{3} / R_{0}^{3}
$$

and in place of $t$ the dimensionless variable

$$
u=\left(\alpha / R_{0}^{4}\right) \int_{0}^{z} R^{4}(y) d y
$$

where the constant $\alpha$ is defined as

$$
\alpha=\left(2 \sigma / \rho R_{0}^{3}\right)^{\frac{1}{2}}
$$

and has the dimensions of reciprocal time. Equation (15) then is transformed to

$$
\frac{1}{6 p^{\prime}} \frac{d}{d u}\left(p^{7 / 3} p^{\prime 2}\right)=1-\frac{1}{p^{1}}-\mu \int_{0}^{u} \frac{p^{\prime}(v) d v}{(u-v)^{\frac{1}{3}}}
$$

in which $p^{\prime}=d p / d u$ and the dimensionless parameter $\mu$ is given by

$$
\mu=\frac{A L \rho^{\prime}}{3 k R_{0 \alpha}}\left(\frac{D}{\pi \alpha}\right)^{\prime}
$$

The physical quantities of interest are now expressed as follows:

$$
\begin{aligned}
t & =\frac{1}{\alpha} \int_{0}^{u} \frac{d v}{p^{4 / 3}(v)} ; \\
R & =R_{0} p^{\dagger} \\
R & =\left(\alpha R_{0} / 3\right) p^{3} p^{\prime} \\
T-T_{0} & =-\frac{\alpha^{2} R_{0}^{2} \mu}{A} \int_{0}^{u} \frac{p^{\prime}(v) d v}{(u-v)^{1}}
\end{aligned}
$$

The values of the radius $R$ for which experimental observations are readily obtainable are much greater than $R_{0}$ so that the asymptotic solution of Eq. (17) $(p \gg 1)$ is of the greatest physical interest. It is of importance, however, to examine the initial stages of the bubble growth since a qualitative description of this portion of the problem is necessary for an understanding of the asymptotic solution. It will be shown that the solution in the asymptotic range is not affected by the details of the mathematical model used to describe the behavior of the bubble for $R$ near $R_{0}$ so that the uncertainty in the surface energy for small values of $R$ causes no difficulties in the physical applications to be made here.

\section{A. Initial Growth of the Bubble}

It is evident from Eq. (17) that a bubble of radius $R_{0}(p=1)$ initially at rest $\left(p^{\prime}(0)=0\right)$ will remain at rest in unstable equilibrium. 'The present mathematical model requires some change in the external conditions before bubble growth will be initiated.

The particular mechanism which will be considered here is a rise in the temperature of the liquid. Suppose, for example, that there is a constant heat source of strength $a$ per unit volume so that the amount of heat added per unit volume $\eta$ from $t=0$ to $t=t$ is

$$
\eta=a t .
$$

Then, if the temperature of the bulk liquid is $T_{0}$ at $t=0$, at time $t$ it will be

$$
T_{\infty}=T_{0}+\eta / \rho c=T_{0}+\eta D / k .
$$

The heat flow problem may be solved ${ }^{6}$ for the boundary conditions of Eqs. (21) and (10), and the temperature at the bubble wall is given by replacing $T_{0}$ in Eq. (12) by $T_{\infty}$. In place of Eq. (19d) one has

$$
T=T_{0}+\eta D / k-\frac{\alpha^{2} R_{0}^{2} \mu}{A} \int_{0}^{u} \frac{p^{\prime}(v) d v}{(u-v)^{\frac{2}{2}}} .
$$

The extension of Eq. (17) to include the heat source term is thus

$$
\frac{1}{6 p^{\prime}} \frac{d}{d u}\left(p^{7 / 3} p^{\prime 2}\right)=1-\frac{1}{p^{1}}-\mu \int_{0}^{u} \frac{p^{\prime}(v) d v}{(u-v)^{\frac{1}{2}}}+\frac{A D}{\alpha^{2} R_{0}^{2} k} \eta .
$$

For values of $p$ near the initial value of unity, one may write

$$
\eta=a t=-\frac{a}{\alpha} \int_{0}^{u} \frac{d v}{p^{4 / 3}(v)} \approx \underset{\alpha}{-}-u,
$$

where the substitution for $t$ follows from Eq. (19a). Equation (23a) now becomes

$$
\frac{1}{6 p^{\prime}} \frac{d}{d u}\left(p^{7 / 3} p^{\prime 2}\right)=1-\frac{1}{p^{1}}-\mu \int_{0}^{u} \frac{p^{\prime}(v) d v}{(u-v)^{\frac{1}{2}}}+\gamma u,
$$

where

$$
\gamma=A D a / \alpha^{3} R_{0}^{2} k .
$$

The constant $\gamma$ is extremely small; for example, in water with a temperature rise of $1^{\circ} \mathrm{C} / \mathrm{sec}$ and $R_{0}=10^{-3}$ $\mathrm{cm}, \gamma$ is approximately $10^{-6}$. The smallness of the constant $\gamma$ implies that the forced growth away from the unstable equilibrium point $p=1, p^{\prime}=0$ is very slow until the bubble radius has increased sufficiently for the surface tension to be partially "relaxed." This initial slow growth is a delay period in the bubble growth, for the bubble radius changes very little until the remaining terms on the right side of Eq. (23b) become appreciable. The bubble growth is then so rapid that the change in the bulk temperature of the liquid is insignificant, and the term in $\gamma$ may be neglected. The delay period effectively gives an initial value of $R$ slightly greater than $R_{0}$ from which the important growth begins. An approximate solution may be found for this initial period of forced growth from the equilibrium point by linearizing Eq. (23b); that is, by neglecting terms of the second order in $p-1$ and its derivatives. The details of this 
TABLE II. Values of the parameters for water at various superheat temperatures. The external pressure $P_{0}=1$ atmos; the constant $A$ has been given the value $4.08 \times 10^{4}$ dynes $\mathrm{cm} /\left({ }^{\circ} \mathrm{C} \mathrm{g}\right)$.

\begin{tabular}{llcccc}
\hline \hline$T_{0}$ & $102^{\circ}$ & $103^{\circ}$ & $104^{\circ}$ & $105^{\circ}$ & $106^{\circ}$ \\
$R_{0}$ & $1.558 \times 10^{-3}$ & $1.019 \times 10^{-3}$ & $7.507 \times 10^{-4}$ & $5.901 \times 10^{-4}$ & $4.832 \times 10^{-4}$ \\
$\alpha$ & $1.797 \times 10^{5}$ & $3.391 \times 10^{5}$ & $5.356 \times 10^{5}$ & $7.677 \times 10^{5}$ & $1.035 \times 10^{6}$ \\
$\mu$ & $5.598 \times 10^{-1}$ & $3.411 \times 10^{-1}$ & $2.407 \times 10^{-1}$ & $1.841 \times 10^{-1}$ & $1.480 \times 10^{-1}$ \\
$b_{1}$ & $-1.073 \times 10^{-1}$ & $-9.099 \times 10^{-1}$ & $-8.101 \times 10^{-1}$ & $-7.409 \times 10^{-1}$ & $-6.890 \times 10^{-1}$ \\
$b_{2}$ & $-4.709 \times 10^{-1}$ & $-4.624 \times 10^{-1}$ & $-7.122 \times 10^{-1}$ & -1.334 & -2.506 \\
$b_{3}$ & $2.339 \times 10^{-1}$ & $-1.481 \times 10^{-3}$ & $-3.586 \times 10^{-1}$ & $-9.725 \times 10^{-1}$ & -1.972 \\
$b_{4}$ & $-5.534 \times 10^{-1}$ & $1.258 \times 10^{-4}$ & $-1.970 \times 10^{-1}$ & -4.598 & $-2.510 \times 10^{1}$ \\
$b_{5}$ & -1.775 & $3.064 \times 10^{-1}$ & 4.298 & $1.850 \times 10^{1}$ & $6.584 \times 10^{1}$ \\
$b_{6}$ & $-7.423 \times 10^{-1}$ & $-2.670 \times 10^{-1}$ & $6.166 \times 10^{-1}$ & $-4.736 \times 10^{-1}$ & $-4.865 \times 10^{1}$ \\
\hline \hline
\end{tabular}

calculation will not be presented here; but, as would be expected, the forced growth away from the equilibrium has an exponential behavior,

$$
R \approx R_{0}\left\{1+\frac{2 \gamma}{\beta\left(3 \beta^{2}+1\right)} e^{\alpha \beta t}\right\} .
$$

The temperature at the bubble wall is given approximately by

$$
T \approx T_{\infty}-\frac{R_{0}^{2} \alpha^{2}}{A} \frac{2 \gamma\left(1-\beta^{2}\right)}{\beta\left(3 \beta^{2}+1\right)} e^{\alpha \beta t} .
$$

The constant $\beta$ is that root of the equation

$$
\beta^{2}+3 \mu(\pi \beta)^{2}-1=0
$$

for which $\beta^{3}$ is positive.

\section{B. Growth Behavior for $R \gg R_{0}$}

After the bubble growth has been initiated, there is a rapid rise in the velocity $\dot{R}$ until the cooling effect becomes important. The bubble wall velocity decreases continuously thereafter. No observations on bubble growth in this region are as yet available and the details of this analysis will be omitted. Of present interest is the asymptotic phase of the bubble growth determined by Eq. (17) which is characterized by the limiting effect on $\dot{R}$ of the heat diffusion from the liquid to the vapor. As $R$ increases, the temperature at the bubble wall decreases steadily, but it cannot fall below $T_{b}$; for, if the vapor temperature fell below $T_{b}$, the pressure difference $p_{v}-P_{0}$ would become negative and the bubble growth would be arrested and eventually reversed. Such behavior is excluded on physical grounds. It therefore follows that the integral on the right-hand side of Eq. (17), which is proportional to the temperature drop at the bubble wall, must approach a limit as $t$ or $u \rightarrow \infty$. A further physical argument determines more precisely the asymptotic behavior of this integral. The left-hand side of Eq. (17) represents essentially the acceleration effects of the bubble growth in the liquid. As the bubble grows, this acceleration tends toward zero because of the cooling effect. It therefore follows that

$$
\mu \int_{0}^{u} \frac{p^{\prime}(v) d v}{(u-v)^{\frac{3}{3}}} \sim 1 \text { as } u \rightarrow \infty .
$$

This asymptotic relation may be inverted to yield ${ }^{8}$

$$
p(u) \approx(2 / \pi \mu) u^{\frac{1}{2}} \text { as } u \rightarrow \infty .
$$

If this result is substituted in Eq. (17), it may be verified that Eqs. (17), (27), and (28) are, in fact, consistent.

Equation (28) is not yet useful since it provides no means of matching the indicated asymptotic solution of Eq. (17) with a solution valid for small values of $p$. The possibility of matching solutions depends on the possibility of shifting the asymptotic solution in $t$ (or in $u$ ) so as to account for the delay period in bubble growth. It is necessary that one be free to shift the asymptotic solution since the duration of the delay period depends completely on the choice of the heat source term while the subsequent behavior of the bubble is independent of this term. A means for making an arbitrary time shift is furnished by noting that, in addition to the asymptotic solution of Eq. (28), Eq. (17) also possesses the solution $p(u)=1$. It will therefore be assumed that the asymptotic solution is described by

$$
\begin{gathered}
p(u)=1, \quad 0<u \leq u_{1}, \\
\mu \int_{u_{1}}^{u} \frac{p^{\prime}(v) d v}{(u-v)^{\frac{1}{2}}}=1-\frac{1}{p^{1}}-\frac{1}{6 p^{\prime}} \frac{d}{d u}\left(p^{7 / 3} p^{\prime 2}\right), \quad u>u_{1} .
\end{gathered}
$$

From Eq. (19a), one has corresponding to Eqs. (29) and (30)

$$
t=\frac{1}{\alpha} \int_{0}^{u} \frac{d v}{p^{4 / 3}(v)}=\left\{\begin{array}{l}
u, \quad 0<u \leq u_{1}, \\
\frac{-}{\alpha}+\frac{1}{\alpha} \int_{u_{1}}^{u} \frac{d v}{p^{4 / 3}}, \quad u>u_{1},
\end{array}\right.
$$

so that $u_{1} / \alpha$ represents the duration of the delay period in the growth. The time delay may be introduced explicitly in the asymptotic solution for $u>u_{1}$ by use of the fact that, if $p(u)$ is a solution of Eq. (30), $p\left(u+u_{0}\right)$ is also a solution where $u_{0}$ is a constant. A consistent scheme for continuing the asymptotic solution may then

Equation (27) is multiplied by $(z-u)^{-1}$ and integrated from $u=0$ to $u=z$ to give Eq. (28). 


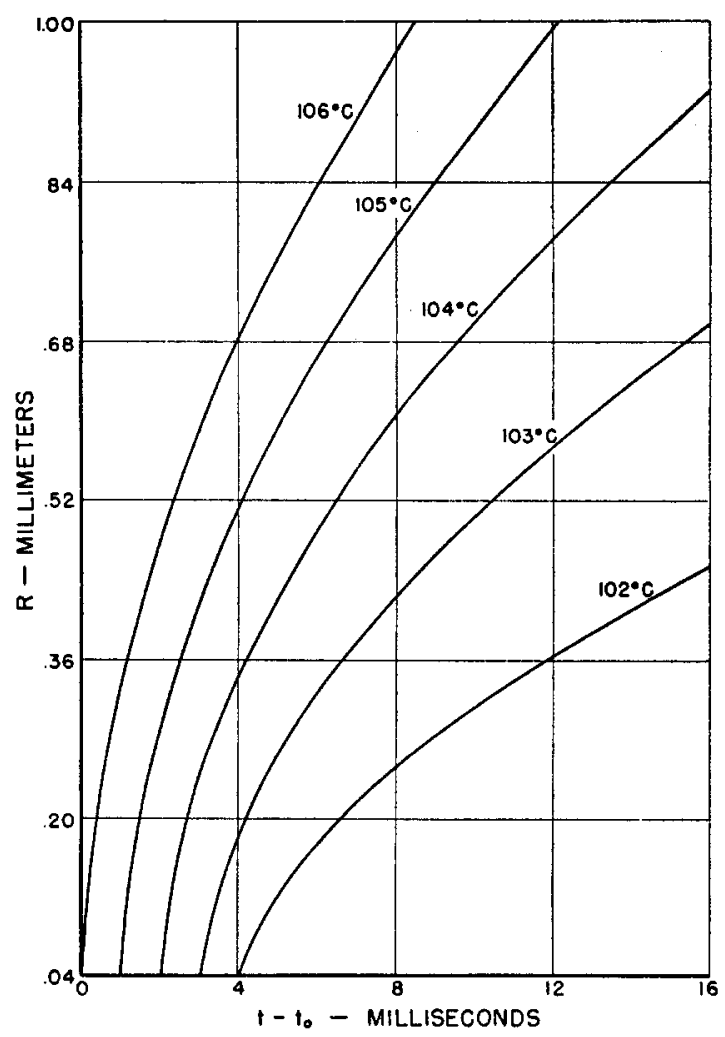

FIG. 1. Calculated bubble radius versus time curves at the indicated superheat temperatures in water at 1 atmos.

be found by taking the solution to be of the form

$$
\begin{gathered}
p(u)=1, \quad 0<u \leq u_{1}, \\
p(u) \sim \frac{2}{\pi \mu}\left(u-u_{0}\right)^{\frac{1}{2}}\left\{1+\frac{b_{1}}{\left(u-u_{0}\right)^{1 / 6}}+\cdots\right. \\
\left.+\frac{b_{5}}{\left(u-u_{0}\right)^{5 / 6}}+\frac{b_{6} \ln \left(u-u_{0}\right)}{\left(u-u_{0}\right)}\right\}, \quad u>u_{1}
\end{gathered}
$$

to seven terms. ${ }^{9}$ When the coefficients $b_{k}$ have been determined, the difference $\left(u_{1}-u_{0}\right)$ is fixed by the requirement that $p\left(u_{1}\right)=1$. The delay period is then determined by the choice of $u_{0}$.

When (32b) is substituted in the integral on the left side of Eq. (30), the result is ${ }^{10}$

$$
\begin{aligned}
& \mu \int_{u_{1}}^{u} \frac{p^{\prime}(v) d v}{(u-v)^{1}} \sim 1+\frac{2 \Gamma(2 / 6)}{3 \pi^{\frac{3}{3}} \Gamma(5 / 6)} \frac{b_{1}}{\left(u-u_{0}\right)^{1 / 6}} \\
& +\frac{\Gamma(1 / 6)}{3 \pi^{\frac{3}{5} \Gamma(4 / 6)}} \frac{b_{2}}{\left(u-u_{0}\right)^{2 / 6}}+\left(\frac{2}{\pi}-b_{3}-\mu\right) \frac{1}{\left(u-u_{0}\right)^{3 / 6}} \\
& -\frac{\Gamma(-1 / 6)}{3 \pi^{\frac{1}{3} \Gamma(2 / 6)}} \frac{b_{4}}{\left(u-u_{0}\right)^{4 / 6}} \\
& \quad-\frac{2 \Gamma(-2 / 6)}{3 \pi^{3} \Gamma(1 / 6)} \frac{b_{5}}{\left(u-u_{0}\right)^{5 / 6}}+2 \frac{b_{6}}{\left(u-u_{0}\right)} .
\end{aligned}
$$

${ }^{9}$ Higher terms are of the form $\left[\ln \left(u-u_{0}\right)\right]^{m} /\left(u-u_{0}\right)^{n / 8}$, where $m$ and $n$ are integers.

${ }^{10}$ See the appendix for the evaluation of the integral.
By Eq. (30), this expression is also asymptotic to

$$
1-\frac{1}{p^{1}}-\frac{1}{6 p^{\prime}} \frac{d}{d u}\left(p^{7 / 3} p^{2}\right) .
$$

If (34) is expanded by (32b), the coefficients of corresponding powers of $\left(u-u_{0}\right)$ may be equated to give a set of successive equations for the parameters $b_{1}, b_{2}, \cdots, b_{6}$. At each step, one has a linear equation for the unknown parameter. A tabulation of these parameters for various superheat conditions in water for an external pressure of 1 atmos is given in Table II.

The leading terms in the asymptotic solution are

$$
\begin{gathered}
p=\left(R / R_{0}\right)^{3} \sim \underset{\pi \mu}{2} u^{\frac{1}{}}\left\{1+0\left(u^{-1 / 6}\right)\right\}, \\
t \sim-\frac{3}{\alpha \mu}\left(\frac{\pi \mu}{2}\right)^{4 / 3} u^{3}\left\{1+0\left(u^{-1 / 6}\right)\right\}, \\
T-T_{0} \sim-\frac{\alpha^{2} R_{0}{ }^{2}}{A}\left\{1+0\left(u^{-1 / 6}\right)\right\} .
\end{gathered}
$$

Thus,

$$
\begin{gathered}
R \sim R_{0}\left(\frac{2}{\pi \mu}\right)\left(\frac{\alpha t}{3}\right)\left\{1+0\left(t^{-3}\right)\right\} \\
T-T_{0} \sim-\frac{\alpha^{2} R_{0}^{2}}{A}\left\{1+0\left(t^{-1}\right)\right\}
\end{gathered}
$$

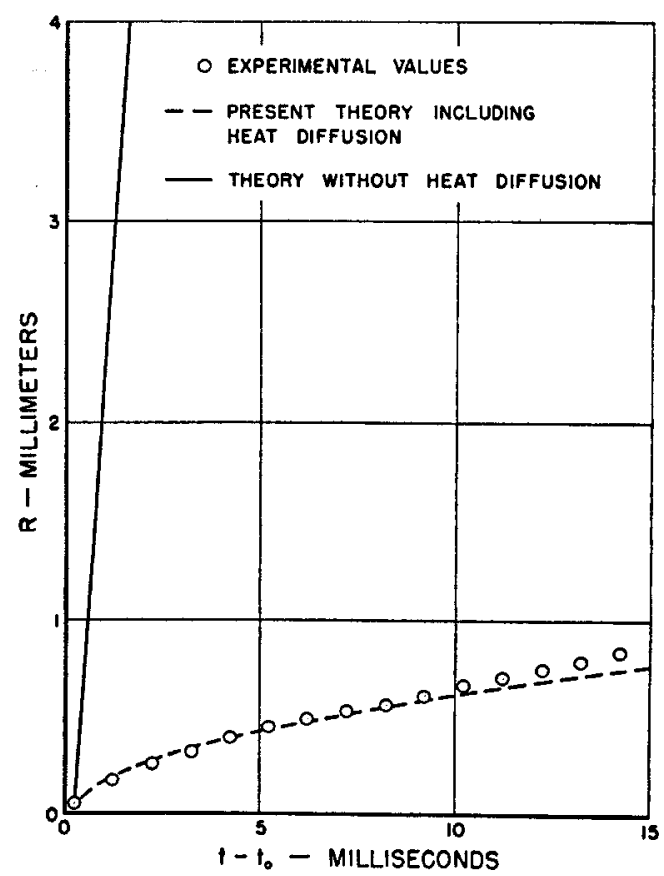

FIG. 2. Comparison of theoretical bubble radius-time values with experimental values in water at 1 atmos with a superheat temperature of $103.1^{\circ} \mathrm{C}$. The solid curve is the Rayleigh growth curve neglecting the cooling effect; the dashed curve includes this effect by the method indicated in the text. 
The vapor density $\rho$ ' may be approximated by a linear function of the temperature over the small range of present concern. Consequently,

$$
\boldsymbol{p}^{\prime} \sim \text { const } \cdot\left\{1+0\left(t^{\frac{1}{2}}\right)\right\} \text { as } t \rightarrow \infty,
$$

by Eq. (36). The volume of the bubble, on the other hand, behaves like

$$
R^{3} \sim \text { const } \cdot t^{\frac{3}{2}}\left\{1+0\left(t^{\frac{1}{2}}\right)\right\} \quad \text { as } t \rightarrow \infty .
$$

It is evident that the vapor density varies slowly in the asymptotic range compared with the bubble volume so that the neglect of $d \rho^{\prime} / d t$ compared with $d R^{3} / d t$ in the formulation of the boundary condition of Eq. (11) is justified.

\section{COMPARISON WITH EXPERIMENT AND CONCLUSION}

With the values of the constants given in Table II, radius-time curves have been computed for water at 1 atmos for superheats in the range of $102^{\circ} \mathrm{C}$ to $106^{\circ} \mathrm{C}$. These curves are presented in Fig. 1. Inasmuch as the delay period may be chosen arbitrarily so far as these asymptotic solutions are concerned, the time scale is determined only within an arbitrary constant which varies from one curve to another. The actual spacing of the curves as presented was chosen so that the time intercepts at $R=0.04 \mathrm{~cm}$ were equally spaced.

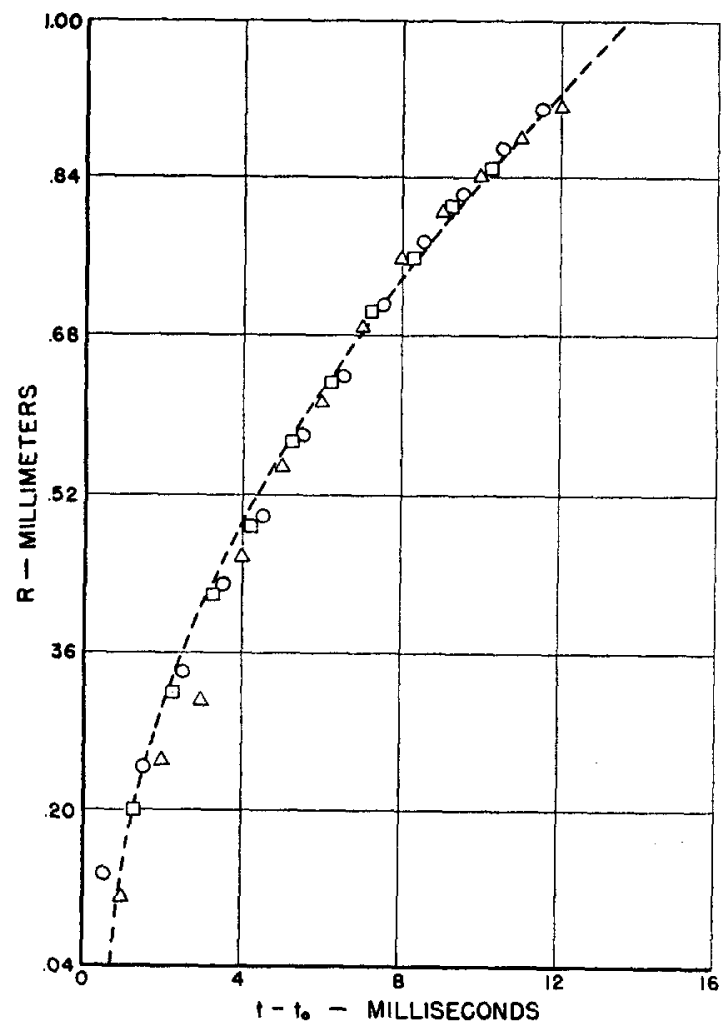

Fig. 3. Comparison of theoretical radius-time values with three sets of experimental values obtained in water superheated to $104.5^{\circ} \mathrm{C}$ at an external pressure of 1 atmos.

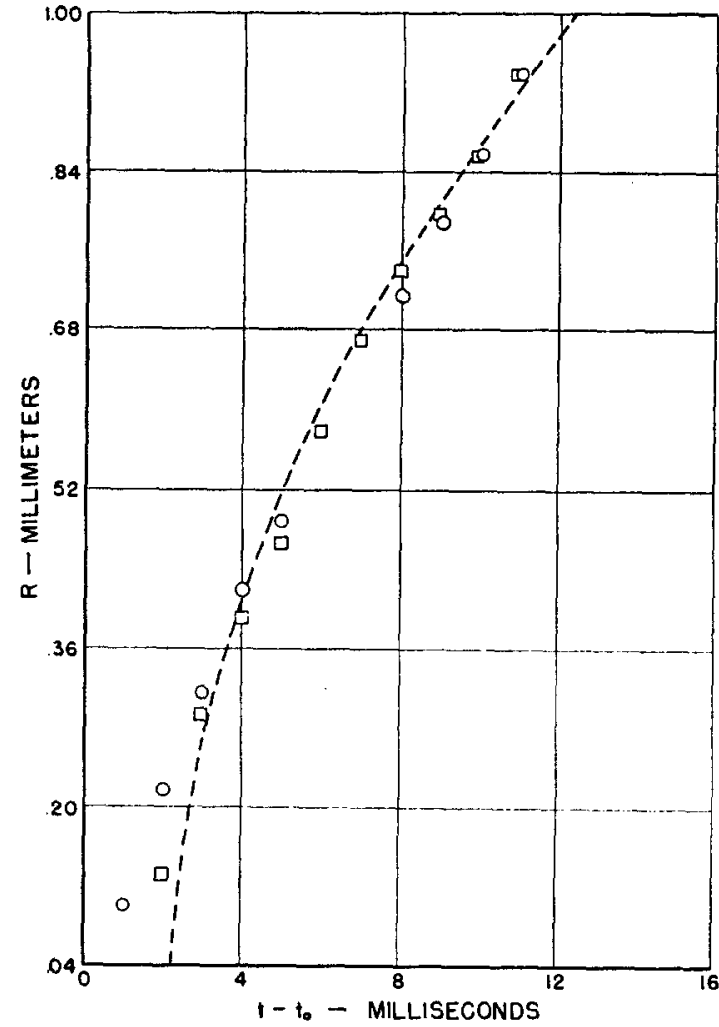

FIg. 4. Comparison of theoretical radius-time values with two sets of experimental values obtained in water superheated to $105.3^{\circ} \mathrm{C}$ at an external pressure of 1 atmos.

Observations have been made by Dergarabedian ${ }^{11}$ on the growth of vapor bubbles in superheated water. The comparison between the theoretical curves and the observed values is shown in Figs. 2, 3, and 4. The theoretical curves were obtained by interpolation from the values graphed in Fig. 1. The time origins for both the theoretical curves and the experimental points are both arbitrary so that a time translation of the theoretical curve has been made in each case to give the best fit. The agreement is, however, seen to be very good. The importance of the cooling effect is evident from Fig. 2 where the Rayleigh solution $[$ Eq. (7)] is also shown.

Equation (35) gives for the leading term in the asymptotic velocity

$$
\dot{R} \sim \underset{\pi \mu}{R_{0}}\left(\frac{\alpha}{3 t}\right)^{\frac{1}{2}}=\left(\frac{3}{\pi}\right)^{\frac{1}{2}} \frac{k\left(T_{0}-T_{b}\right)}{L \rho^{\prime} D^{\frac{1}{2}}} \frac{1}{t^{\frac{1}{2}}} .
$$

Although the number of terms given in Eq. (32b) is sufficient for high accuracy in the range of values of $R$ used in Figs. 1-4, the leading term in Eq. (32b) is in error by approximately 10 to 30 percent for the smallest values of $R$. This leading term is sufficiently simple, however, that a rough physical argument should give the essential variation of Eq. (37). The argument is as

${ }^{11}$ P. Dergarabedian, J. Appl. Mech. (to be published). 
follows: At a time $t$ at which the bubble radius $R$ is much greater than $R_{0}$, the difference between the temperature in the liquid at the bubble wall and that in the liquid at a distance is only slightly less than $T_{0}-T_{b}$. This temperature drop takes place principally in a liquid layer around the bubble of approximate thickness given by the diffusion length $(D t)^{\frac{1}{2}}$. The heat flow into the bubble per unit time is therefore given roughly by

$$
\dot{Q} \approx\left[k\left(T_{0}-T_{b}\right) /(D t)^{\frac{1}{2}}\right] 4 \pi R^{2} .
$$

The heat requirement per unit time of evaporation, on the other hand, is

$$
\dot{Q}=L d / d t\left(4 \pi R^{3} \rho^{\prime} / 3\right) \approx 4 \pi R^{2} \dot{R} L \rho^{\prime} .
$$

When (39) is equated to (38), there results

$$
\dot{R} \sim k\left(T_{0}-T_{b}\right) / L \rho^{\prime}(D t)^{\prime},
$$

which agrees in order of magnitude with the leading term as given by Eq. (37).

Some experiments have recently been performed by Dergarabedian on vapor bubble growth in pure $\mathrm{CCl}_{4}$. At moderate superheats, its vapor pressure curve is approximately parallel to that of water, displaced to lower temperatures. If the rates of bubble growth in these two liquids are compared at the same value of the temperature difference $\left(T_{0}-T_{b}\right)$, they should thus be roughly in the same ratio as $k /\left(L \rho^{\prime} D^{1}\right)$ for the two liquids. This constant is about 3.5 greater in water than in carbon tetrachloride. Dergarabedian's observations on bubble growths are in good agreement with this value.

\section{APPENDIX}

\section{Evaluation of the Temperature Integral [Eq. (33)]}

By differentiating $p(u)[$ Eq. (32b)] and substituting into the temperature integral there results, after a change of variable,

$$
\begin{gathered}
\mu \int_{u_{1}}^{u} \frac{p^{\prime}(v) d v}{(u-v)^{\frac{1}{2}}} \\
\sim \frac{d v}{\pi} \int_{\left(u_{1-u}-u_{0} /\left(u-u_{0}\right)\right.}^{1} \frac{v^{\frac{1}{2}}(1-v)^{\frac{1}{2}}}{1}+\left[\frac{2}{3} \frac{b_{1}}{\left(u-u_{0}\right)^{1 / 6}}\right]_{v^{1 / 6}}^{1} \\
+\left[\frac{1}{3} \frac{b_{2}}{\left(u-u_{0}\right)^{2 / 6}}\right] \frac{1}{v^{2 / 6}}-\left[\frac{1}{3} \frac{b_{4}}{\left(u-u_{0}\right)^{4 / 6}}\right] \frac{1}{v^{4 / 6}} \\
-\left[\frac{b_{5}}{3} \frac{1}{\left(u-u_{0}\right)^{5 / 6}}\right] \frac{1}{v^{5 / 6}} \\
\left.-\left[\frac{b_{6}}{u-u_{0}}\right]\left[\ln v+\ln \left(u-u_{0}\right)-2\right]_{v}^{1}\right\} .
\end{gathered}
$$

Consider a typical integral appearing in (a),

$$
I_{s}(x)=\int_{x}^{1} \frac{c^{s-1} d v}{(1-v)^{\frac{1}{2}}} .
$$

For $0<x<1, \operatorname{Re}(s)>0$, this is the hypergeometric function ${ }^{12}$

$$
\begin{aligned}
I_{s}(x)= & -\frac{1}{s}\left[F\left(\frac{1}{2}, s ; s+1 ; 1\right)-x^{s} F\left(\frac{1}{2}, s ; s+1 ; x\right)\right], \\
& \sim \frac{\Gamma\left(\frac{1}{2}\right) \Gamma(s)}{\Gamma\left(s+\frac{1}{2}\right)}-\frac{1}{s} \text { as } x \rightarrow 0^{+} .
\end{aligned}
$$

By an argument based on the theory of analytic continuation, however, it may readily be shown that (c) is valid provided only that $0<x<1, s \neq 0,-1,-2, \cdots$, and hence that (d) holds for $0<x<1, \operatorname{Re}(s)>-1$. For $s=0$, (d) is meaningless, but gives a correct result under a limiting procedure. By differentiating (d) with respect to $s$ at $s=-\frac{1}{2}$, one readily finds

$$
\int_{x}^{1} \frac{\ln v d v}{v^{1}(1-v)^{\frac{1}{2}}} \sim-2 \pi+\frac{2}{x^{\frac{1}{3}}}(\ln x+2) \text { as } x \rightarrow 0^{+} .
$$

With the aid of (d), (e), Eq. (a) therefore becomes

$$
\begin{gathered}
\sim\left\{1+0.89266 \frac{b_{1}}{\left(u-u_{0}\right)^{1 / 6}}+0.77306 \frac{p^{\prime}(v) d v}{(u-v)^{\frac{3}{3}}}\right. \\
+0.47545 \frac{b_{4}}{\left(u-u_{0}\right)^{2 / 6}}+0.27450 \frac{b_{5}}{\left(u-u_{0}\right)^{5 / 6}} \\
\left.+2 \frac{b_{6}}{\left(u-u_{0}\right)}\right\}-\frac{2}{\pi} \frac{1}{\left(u-u_{0}\right)^{\frac{1}{2}}}\left(u_{1}-u_{0}\right)^{3 / 6} \\
\times\left\{1+\frac{b_{1}}{\left(u_{1}-u_{0}\right)^{1 / 6}}+\frac{b_{2}}{\left(u_{1}-u_{0}\right)^{2 / 6}}+\frac{b_{4}}{\left(u_{1}-u_{0}\right)^{4 / 6}}\right. \\
\left.+\frac{b_{5}}{\left(u_{1}-u_{0}\right)^{5 / 6}}+b_{6} \frac{\ln \left(u_{1}-u_{0}\right)}{u_{1}-u_{0}}\right\}
\end{gathered}
$$

after a rearrangement, several terms cancelling. The $\Gamma$ functions appearing in (d) have been evaluated numerically in (f). By comparison with the asymptotic form for $p(u)$ of Eq. (30), the last term in (f) may be written

$$
1 /\left(u-u_{0}\right)^{\frac{1}{1}}\left\{(2 / \pi) b_{3}-\mu p\left(u_{1}\right)\right\} .
$$

Inasmuch as $p\left(u_{1}\right)=1$ by assumption, Eq. (f) reduces to Eq. (33) of the text.

12 E. T. Copson, Introduction to Theory of Functions of a Complex Variable (Oxford University Press, London, 1935), pp. 249, 251. 